Tropical Journal of Pharmaceutical Research February 2020; 19 (2): 227-232

ISSN: $1596-5996$ (print); 1596-9827 (electronic)

(C) Pharmacotherapy Group, Faculty of Pharmacy, University of Benin, Benin City, 300001 Nigeria.

\title{
Epigallocatechin gallate activates miR-193a-3p and protects mice against glucocorticoid-induced osteoporosis by targeting NFATC1 expression
}

\author{
Ben Dou, Xiaohui Wu, Yisong Xie, Hongliang Ruan, Xiaolan Liu* \\ Department of Orthopedics, The Second Affiliated Hospital of Hunan University of Chinese Medicine, Changsha, Hunan \\ 410005, China \\ *For correspondence: Email: onorouj@yahoo.com; Tel: 0086-13627489118
}

Sent for review: 20 August 2019

Revised accepted: 24 January 2020

\begin{abstract}
Purpose: To investigate the effect of epigallocatechin gallate (EGCG) on microRNAs in a mouse model of glucocorticoid-induced osteoporosis (GIOP), and the mechanism involved.

Methods: Osteoclast-specific marker mRNA expressions, receptor activator of nuclear factor kappa-B ligand (RANKL), receptor activator of nuclear factor $\kappa B$ (RANK), and miRNA expressions were determined using reverse transcription polymerase chain reaction (RT-qPCR) analysis. Western blotting was used to assay protein expressions, while miRNA and 3'UTR interaction studies were carried out with reporter assay.

Results: Treatment with EGCG resulted in downregulation of glucocorticoid-induced expressions of RANKL, RANK and osteoclast-specific markers i.e. tumor necrosis factor receptor-associated factor 6 , (TRAF6), nuclear factor of activated T cells 1 (NFATc1), cathepsin K, matrix metallopeptidase 9 (MMP9) and tartrate-resistant acid phosphatase (TRAP). Furthermore, EGCG treatment significantly reduced reactive oxygen species (ROS) levels and inflammatory cytokine expressions in GIOP mice. The expression of miRNA-targeting osteoclast marker mmu-mir-193-3p was significantly down-regulated in GIOP mice. However, EGCG treatment increased mmu-mir-193-3p expression and had specific interaction with NFATC1 3'UTR (3'-untranslated region). In vitro results showed that mmu-mir-193-3p mimics downregulated dexamethasone (DXM)-induced osteoclast-specific marker expressions.

Conclusion: These results show that EGCG exerts a protective role against GIOP by upregulating miR193a-3p expressions.
\end{abstract}

Keywords: Epigallocatechin gallate, Glucocorticoids, RANKL, Osteoporosis

\begin{abstract}
This is an Open Access article that uses a fund-ing model which does not charge readers or their institutions for access and distributed under the terms of the Creative Commons Attribution License (http://creativecommons.org/licenses/by/4.0) and the Budapest Open Access Initiative (http://www.budapestopenaccessinitiative.org/read), which permit unrestricted use, distribution, and reproduction in any medium, provided the original work is properly credited.

Tropical Journal of Pharmaceutical Research is indexed by Science Citation Index (SciSearch), Scopus, International Pharmaceutical Abstract, Chemical Abstracts, Embase, Index Copernicus, EBSCO, African Index Medicus, JournalSeek, Journal Citation Reports/Science Edition, Directory of Open Access Journals (DOAJ), African Journal Online, Bioline International, Open-J-Gate and Pharmacy Abstracts
\end{abstract}

\section{INTRODUCTION}

Glucocorticoid (GC) drugs are used to reduce immune responses during organ transplantation and inflammatory diseases [1]. Post-GC therapy patients develop various complications such as obesity, diabetes mellitus, cardiovascular disease and bone loss [2]. Clinical studies have revealed risk of asthma in $50 \%$ of patients who use GCs [3]. Glucocorticoids (GCs) induce osteoblast apoptosis, and target osteoclast bone resorptive activity, ultimately mediating 
osteoporosis. In the presence of receptor activator of RANKL and macrophage-colony stimulating factor (M-CSF), osteoclasts differentiate and fuse to form multinuclear osteoclasts which function in bone resorptive activity [4]. In this study, the role of miRNAs in GC-induced osteoporosis, and the protective effect of the antioxidant EGCG, were investigated. Epigallocatechin gallate (EGCG) possesses antioxidant property, scavenges free radicals and reduces oxidative stress [5]. The bioactivity of EGCG is due toits eight hydroxyl groups and gallate moiety [6]. It has been shown that EGCG treatment reduces high fat dietinduced weight gain [7], and regulates blood glucose and lipid levels [8].

Deregulated miRNA expressions are the prime causes of disease [9]. This study was aimed at investigating the miRNA expressions and the protective effect of EGCG against GIOPmediated miRNA expressions in mice.

\section{EXPERIMENTAL}

\section{Animals and treatment}

Twenty-four 8 week-old, male C57BL/6J mice were purchased from Research Center at The Second Affiliated Hospital of Hunan University of Chinese Medicine, China. The mice had a mean weight of $35 \pm 1.3 \mathrm{~g}$. Approval for the study was received from ethical committee of The Second Affiliated Hospital of Hunan University of Chinese Medicine, China (approval no. 8673/UN6.HUCM /EC/2017). The animal experiment was performed in line with the guidelines of the Association for the Assessment and Accreditation of Laboratory Animal Care International (AAALAC) [10].

The mice were allowed 1-week acclimatization to laboratory environment at a temperature of $22 \pm$ $2{ }^{\circ} \mathrm{C}$ and relative humidity of $50-52 \%$. The animals were divided into four groups: sham, EGCG, GIOP and GIOP+EGCG, with 6 mice per group. Glucocorticoid-induced osteoporosis was induced by treating the mice with dexamethasone (DXM) at a dose of $10 \mathrm{mg} / \mathrm{kg}$ intramuscularly for 10 weeks (3 times/per week). Mice in the sham group received vehicle during the treatment period, while mice in the EGCG group were given EGCG orally at a dose of 100 $\mathrm{mg} / \mathrm{kg}$ daily for 10 weeks.

\section{Measurement of reactive oxygen species}

The tissue levels of ROS were determined using ROS detection kit (Abcam- ab113851), as per manufacturer's instructions.

\section{Enzyme linked immunosorbent assay (ELISA) for serum cytokines}

Serum cytokines (IL-6 and TNF- $\alpha$ ) were assayed using ELISA kits, as per instructions in the kit manual.

\section{Quantitative reverse transcription PCR (RT- qPCR)}

Total tissue RNA was isolated using Trizol reagent. The RNA was converted into CDNA and the expressions of RANKL, RANK, TRAF6, NFATc1, Cathepsin K, MMP9 and TRAP were determined using RT-qPCR. Their expressions were calculated relative to that of $\beta$-actin which served as control.

\section{Target gene prediction and miRNA RT-qPCR}

TargetScan 6.2 (http://targetscan.org) was used to predict miRNA targeting site on the osteoclast markers NFATc1, TRAF6 and RANKL gene. Tissue miRNA was isolated using miRNA isolation kit (Qiagen, USA) as per manufacturer's instructions. Then, the expressions of mmu-miR193a-3p, mmu-miR-124-3p, mmu-miR-17-3p and mmu-miR-20a-5p were determined using TaqMan miRNA assays as per manufacturer's instructions.

\section{Cell culture}

RAW 264.7 cells were stimulated with M-CSF and RANKL in the differentiation medium. To identify the effect of DXM on OC differentiation, 150nM DXM was used. Cells were transfected with 50nM miR-193a-3p mimics in DXM+miR193a-3p group.

\section{Transfection of MiR-193-3p mimics}

Overexpression of MiR-193a-3p over was achieved using miRIDIAN miR-193a-3p mimics (Dharmacon). The miR-193-3p mimics were added using Lipofectamine 2000 transfection protocol, and were used for reporter assay (after $24 \mathrm{~h}$ ), or for determination of mimics-mediated osteoclast differentiation.

\section{Reporter assay}

Following overnight attachment, the cells were transfected with 50nM miR-193-3p mimics/Scramble and $1 \mu \mathrm{g}$ of WT/MT NFATc1 3'UTR for $24 \mathrm{~h}$. Reporter assay was carried out using Dual-Luciferase Reporter Assay System (Promega).

\section{Western blot}


The cells were lysed using RIPA lysis buffer. Then, $50 \mu \mathrm{l}$ of protein was subjected to gradient SDS-polyacrylamide gel electrophoresis and transferred to PVDF membrane. Then, the membrane was incubated overnight with primary antibodies for TRAF6, NFATc1, cathepsin K, MMP9 and TRAP at $4{ }^{\circ} \mathrm{C}$. The membrane was washed thrice with TBST, and incubated with horse radish-conjugated secondary antibody $(1: 1000)$ for $1 \mathrm{~h}$ at room temperature. The blots were visualized using enhanced chemiluminescence $(E C L)$, and the images were analyzed.

\section{Statistical analysis}

The results are expressed as mean \pm standard deviation. Each experiment was conducted in triplicate. Statistical differences were analyzed with analysis of variance, using Prism6 (GraphPad Software Inc). Values of $p<0.001$ were taken as indicative of statistical significance.

\section{RESULTS}

\section{EGCG reduced GIOP by regulating osteoclast marker expressions}

Figure $1 \mathrm{~A}$ shows significant upregulation of the expressions of RANK and RANKL in GIOP mice model, when compared to sham mice. However, EGCG treatment downregulated the expressions of RANKL and RANK, when compared to GIOP mice. Furthermore, the upregulated osteoclastspecific mRNA and protein expressions (TRAF6, NFATc1, MMP9, Cathepsin K and TRAP) in GIOP mice group were significantly downregulated in EGCG-treated GIOP mice group. These results show that EGCG exerted protective effect against glucocorticoid-induced osteoporosis.

\section{EGCG prevented oxidative stress and inflammation}

Figure 2 A shows that GIOP mice had significantly upregulated oxidative stress, with increased ROS levels, when compared to sham mice. However, EGCG treatment reduced the oxidative stress in GIOP mice. Furthermore, the GIOP-induced increases in inflammatory cytokine expressions (TNF- $\alpha$ and IL-6) were significantly reduced by EGCG treatment. These results are shown in Figure $2 \mathrm{~B}$.
A
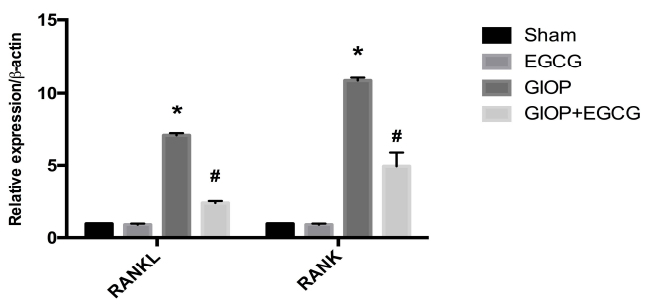

B

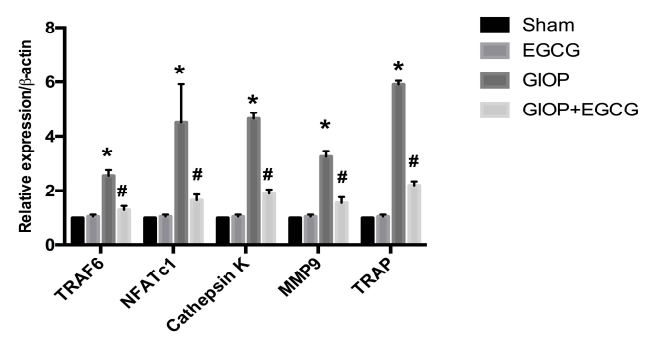

C

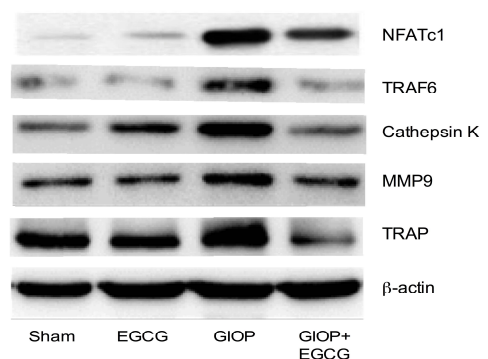

Figure 1: EGCG down-regulates osteoclast marker expressions in GIOP mice. Effect of EGCG on (A) RANKL, RANK mRNA expressions, (B) Osteoclast marker expressions (mRNA) - TRAF6, NFATc1, cathepsin K, MMP9, TRAP;(C) Osteoclast marker TRAF6, NFATc1, Cathepsin K, MMP9, TRAP protein expressions. ${ }^{*} p<0.001$ compared to sham mice. $\# p<0.001$ compared to GIOP mice

A

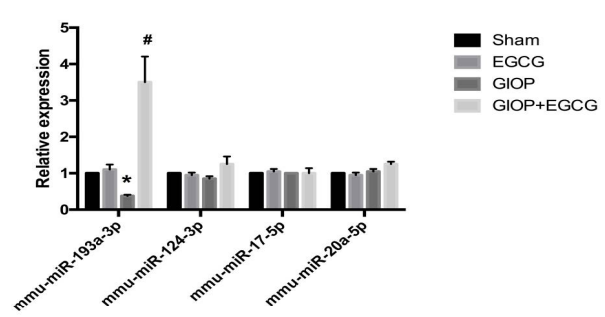

B

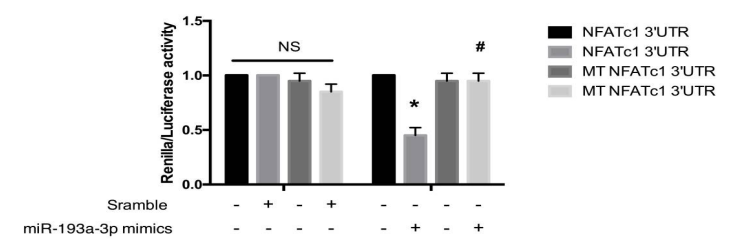

Figure 2: Effect of EGCG on oxidative stress and inflammation in GIOP mice. (A) EGCG reduced ROS levels in GIOP mice. (B) Glucocorticoid-stimulated inflammatory cytokine levels were attenuated by EGCG. The results are expressed in $\mathrm{pg} / \mathrm{mL}$; ${ }^{*} p<0.001$, compared to sham mice; $\# p<0.001$, compared to GIOP mice 


\section{Effect of glucocorticoid and EGCG on miRNA levels}

In order to determine whether miRNA expressions regulate osteoporosis, the expressions of mmu-miR-193a-3p, mmu-miR124-3p, mmu-miR-17-3p, and mmu-miR-20a5ptargetingNFATc1, TRAF6 and RANKL, respectively were assayed. The expression of mmu-miR-193a-3p was significantly reduced in GIOP mice, when compared to sham mice. However, the miR-193a-3p expressions were significantly increased in GIOP mice treated with EGCG. Reporter assay showed that miR-193a$3 p$ had potential interaction with WT 3'UTR of NFATc1 mRNA, whereas MT 3'UTR of NFATc1 and miR-193a-3p had no interaction. These results show that the EGCG-mediated protective effect was exerted through upregulated miR193a-3p expression (Figure 3).

A

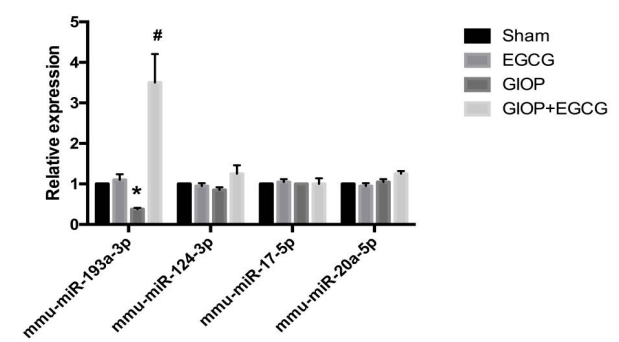

B

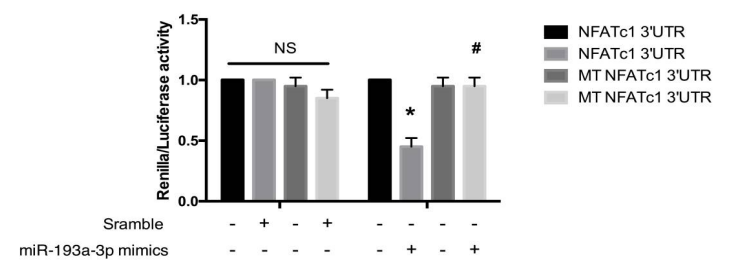

Figure 3: Effect of EGCG on miR-193a-3p expression and NFATc1. (A) Effect of glucocorticoids and EGCG on miRNA expressions (miR-193a-3p, miR-124-3p, miR-17-5p, and miR-20a-5p). (B) Reporter assay showing interaction between miR-193a-3p and NFATc1 3'UTR. ${ }^{*} P<0.001$, compared to sham mice; $\# p<0.001$, compared to GIOP mice

\section{MiR-193a-3p regulated osteoclast-specific markers}

In vitro treatment with miR193a-3p mimics significantly downregulated the expressions of DXM-induced osteoclast markers (TRAF6, NFATc1, MMP9, Cathepsin K and TRAP) at both mRNA and protein expression levels (Figures 4 $A$ and $B)$.
A

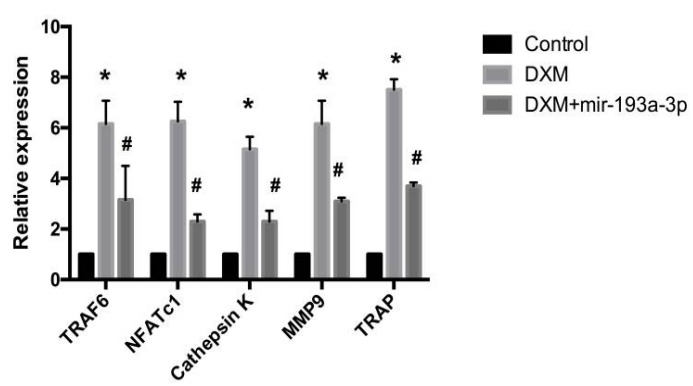

B

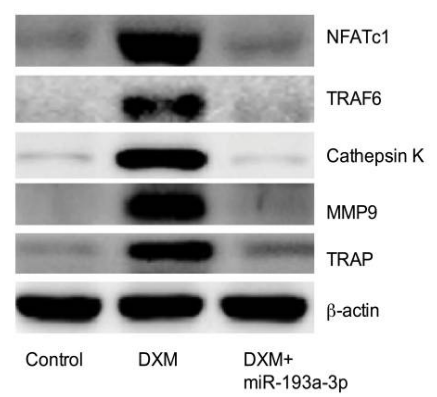

Figure 4: Effect of MiR-193a-3p on expressions of osteoclast-specific markers. MiR-193a-3p mimics downregulated DXM-induced, osteoclast-specific mRNA and protein expressions of TRAF6, NFATc1, cathepsin K, MMP9 and TRAP. ${ }^{*} P<0.001$, compared to sham mice; $\# p<0.001$, compared to GIOP mice.

\section{DISCUSSION}

In this study, EGCG-mediated increases in miR193-3p expression had protective effect against GIOP-induced oxidative stress and osteoclast markers.

Glucocorticoids mediate osteoclast differentiation through coordinated mechanisms involving osteoclasts and osteoblasts [11]. Osteoblasts secrete RANKL which interact with RANK (RANK-RANKL) to initiate osteoclast differentiation. In the present study, the glucocorticoid upregulated the expressions of RANK and RANKL. Moreover, there was significant upregulations of osteoclast-specific marker mRNA and protein expressions in GIOP mice. However, EGCG treatment in GIOP mice resulted in protective effect against osteoclast differentiation through downregulation of RANKL, RANK and osteoclast markers. Previous studies have shown that GIOP increased CTSK and Runx2 expressions in a rat model [12]. Glucocorticoids upregulated RANKL expression in the osteoblasts and promoted osteoclast differentiation in human PBMCs (monocytes) [13]. Moreover, it has been reported that glucocorticoids stimulated osteoclast 
differentiation in mouse calvarial bones [14].

Oxidative stress and inflammatory cytokines are implicated in bone destruction [14-16]. Chronic inflammation is associated with bone resorption. The roles TNF- $\alpha$, IL- $1 \beta$ and IL- 6 [17-20] and oxidative stress [21] in osteoclastogenesis have been reported. In this study, the increases in levels of ROS and inflammatory cytokines due to GIOP were significantly downregulated by EGCG treatment. The reno-protective role of EGCG has been demonstrated in unilateral ureteral obstruction (UUO) mice model through upregulation of Nrf2/HO-1 signaling pathway [22]. Moreover, a study has shown that EGCG attenuated uric acid-mediated inflammatory responses through downregulation of the NOTCH signaling pathway [23].

The miRNA-mediated protein regulation plays a critical role in disease control. Among the various shortlisted miRNAs, mmu-miR-193-3p was significantly downregulated in GIOP, with specific target for NFATc1. This particular miRNA was upregulated by EGCG treatment in GIOP mice. Nuclear factor-activated T cell c1 (NFATc1) is a transcription factor that increases the expressions of the target proteins TRAP and cathepsin K $[24,25]$. Evidence has shown that NFATc1 alone is sufficient for osteoclastogenesis process [24]. Deficiency of NFATc1 prevents osteoclast formation, while overexpression of NFATc1 enhances osteoclastogenesis [24]. From the in vitro studies, miR-193-3p mimics in the presence of DEX treatment significantly downregulated the expressions of NFATc1 and osteoclast markers (TRAF6, cathepsin K, MMP9 and TRAP) at both mRNA and protein levels.

\section{CONCLUSION}

These findings show the protective role of EGCG against GIOP-induced osteoclast marker expressions via miR-198-3p/NFATc1 targeting. Thus, the antioxidant EGCG has promising potential as a therapeutic agent for glucocorticoids-induced osteoporosis.

\section{DECLARATIONS}

\section{Conflict of interest}

No conflict of interest is associated with this work.

\section{Contribution of authors}

We declare that this work was done by the author(s) named in this article and all liabilities pertaining to claims relating to the content of this article will be borne by the authors. Ben Dou, Xiaolan Liu- conceived and designed the study; Xiaohui Wu, Yisong Xie- collected and analyzed the data; Ben Dou, Hongliang Ruan -wrote the manuscript. Xiaolan Liu-Approved final version of the manuscript. All authors read and approved the manuscript for publication.

\section{Open Access}

This is an Open Access article that uses a funding model which does not charge readers or their institutions for access and distributed under the terms of the Creative Commons Attribution License (http://creativecommons.org/licenses/by/ 4.0) and the Budapest Open Access Initiative (http://www.budapestopenaccessinitiative.org/rea d), which permit unrestricted use, distribution, and reproduction in any medium, provided the original work is properly credited.

\section{REFERENCES}

1. Coutinho AE, Chapman KE. The anti-inflammatory and immunosuppressive effects of glucocorticoids, recent developments and mechanistic insights. Mol Cell Endocrinol 2011; 335: 2-13.

2. Vegiopoulos A, Herzig S. Glucocorticoids, metabolism and metabolic diseases. Mol Cell Endocrinol 2007; 275: 43-61.

3. Reid IR. Glucocorticoid osteoporosis-mechanisms and management. Eur J Endocrinol 1997; 137: 209-217.

4. Adler RA. Glucocorticoid-induced osteoporosis: management update. Curr Osteoporos Rep 2010; 8:1014.

5. Lambert JD, Elias RJ. The antioxidant and pro-oxidant activities of green tea polyphenols: a role in cancer prevention. Arch Biochem Biophys 2010; 501:65-72.

6. Niemeyer ED, Brodbelt JS. Isomeric differentiation of green tea catechins using gas-phase hydrogen/deuterium exchange reactions. J Am Soc Mass Spectrom 2007; 18: 1749-1759.

7. Klaus $S$, Pültz $S$, Thone-Reineke $C$, Wolfram $S$. Epigallocatechin gallate attenuates diet-induced obesity in mice by decreasing energy absorption and increasing fat oxidation. Int J Obes (Lond) 2005; 29: 615-623.

8. Roghani M, Baluchnejadmojarad T. Hypoglycemic and hypolipidemic effect and antioxidant activity of chronic epigallocatechin-gallate in streptozotocin-diabetic rats. Pathophysiol 2010: 17: 55-59.

9. Paul P, Chakraborty A, Sarkar D, Langthasa M, Rahman $M$, Bari M, Singha RS, Malakar AK, Chakraborty $S$. Interplay between miRNAs and human diseases. J Cell Physiol 2018; 233: 2007-2018.

10. Gettayacamin $M$, Retnam L. AAALAC International Standards and Accreditation Process. Toxicol Res 2017; 33:183-189.

Trop J Pharm Res, February 2020; 19(2): 231 
11. Jia D, O'Brien CA, Stewart SA, Manolagas SC, Weinstein RS. Glucocorticoids act directly on osteoclasts to increase their life span and reduce bone density. Endocrinol 2006, 147: 5592-5599.

12. Ren $H$, Shen G, Tang J, Qiu T, Zhang Z, Zhao W, Yu X, Huang J, Liang, Yao $Z$, Yang $Z$, Jiang $X$. Promotion effect of extracts from plastrum testudinis on alendronate against glucocorticoid-induced osteoporosis in rat spine. Sci Rep 2017; 7:10617.

13. Sivagurunathan S, Muir MM, Brennan TC, Seale JP, Mason RS. Influence of glucocorticoids on human osteoclast generation and activity. J Bone Miner Res 2005; 20: 390-398.

14. Swanson C, Lorentzon M, Conaway HH, Lerner UH. Glucocorticoid regulation of osteoclast differentiation and expression of receptor activator of nuclear factorkappaB (NF-kappaB) ligand, osteoprotegerin, and receptor activator of NF-kappaB in mouse calvarial bones. Endocrinol 2006; 147: 3613-3622.

15. Hyeon S, Lee $H$, Yang $Y$, Jeong W. Nrf2 deficiency induces oxidative stress and promotes RANKL-induced osteoclast differentiation. Free Radic Biol Med 2013; 65 : 789-799.

16. Wijekoon S, Bwalya EC, Fang J, Kim S, Hosoya K, Okumura M. Chronological differential effects of proinflammatory cytokines on RANKL-induced osteoclast differentiation of canine bone marrow-derived macrophages. J Vet Med Sci 2017; 79: 2030-2035.

17. Srinivasan S, Koenigstein A, Joseph J, Sun L, Kalyanaraman B, Zaidi M, Avadhani NG. Role of mitochondrial reactive oxygen species in osteoclast differentiation. Ann N Y Acad Sci 2010; 1192: 245-252.
18. Baker-LePain JC, Nakamura MC, Lane NE. Effects of inflammation on bone: an update. Curr Opin Rheumatol 2011; 23: 389-395.

19. Brennan FM, Mclnnes IB. Evidence that cytokines play a role in rheumatoid arthritis. J Clin Invest 2008; 118: 3537-545.

20. Walsh NC, Crotti TN, Goldring SR, Gravallese EM. Rheumatic diseases: the effects of inflammation on bone. Immunol Rev 2005; 208: 228-251.

21. Feng YL, Tang XL. Effect of glucocorticoid-induced oxidative stress on the expression of Cbfa1. Chem Biol Interact 2014; 207:26-31.

22. Wang $Y$, Wang B, Du F, Su X, Sun G, Zhou G, Bian $X$, Liu N. Epigallocatechin-3-Gallate Attenuates Oxidative Stress and Inflammation in Obstructive Nephropathy via NF-KB and Nrf2/HO-1 Signalling Pathway Regulation. Basic Clin Pharmacol Toxicol 2015;117: 164-172.

23. Xie $H$, Sun J, Chen Y, Zong M, Li S, Wang Y. EGCG Attenuates Uric Acid-Induced Inflammatory and Oxidative Stress Responses by Medicating the NOTCH Pathway. Oxid Med Cell Longev 2015; 214836.

24. Takayanagi H, Kim S, Koga $T$, Nishina H, Isshiki $M$, Yoshida $H$, Saiura A, Isobe $M$, Yokochi $T$, Inoue J, et al. Induction and activation of the transcription factor NFATc1 (NFAT2) integrate RANKL signaling in terminal differentiation of osteoclasts. Dev Cell 2002; 3: 889-901.

25. Kim K, Kim JH, Lee J, Jin HM, Lee SH, Fisher DE, Kook $H$, Kim KK, Choi Y, Kim N. Nuclear factor of activated $T$ cells c1 induces osteoclast-associated receptor gene expression during tumor necrosis factor-related activation-induced cytokine-mediated osteoclastogenesis. J Biol Chem 2005; 280: 35209-35216. 